\title{
An Evaluation of Complementary Approaches to Elucidate Fundamental Interfacial Phenomena Driving Adhesion of Energetic Materials
}

\author{
Darby J. Hoss, ${ }^{[a]}$ Robert Knepper, ${ }^{[b]}$ Peter J. Hotchkiss, ${ }^{[b]}$ Alexander S. Tappan, ${ }^{[b]}$ \\ Bryan W. Boudouris, ${ }^{[\mathrm{a}]}$ and Stephen P. Beaudoin* ${ }^{[\mathrm{a}]}$
}

\footnotetext{
${ }^{a}$ School of Chemical Engineering, Purdue University, 480 Stadium Mall DriveWest Lafayette, IN 47907-2100, USA

*e-mail: sbeaudoi@purdue.edu

${ }^{\mathrm{b}}$ Sandia National Laboratories, Albuquerque, NM 87185, USA
}

\begin{abstract}
Cohesive Hamaker constants of solid materials are measured via optical and dielectric properties (i.e., Lifshitz theory), inverse gas chromatography (IGC), and contact angle measurements. To date, however, a comparison across these measurement techniques for common energetic materials has not been reported. This has been due to the inability of the community to produce samples of energetic materials that are readily compatible with contact angle measurements. Here we overcome this limitation by using physical vapor deposition to produce thin films of five common energetic materials, and the contact angle measurement approach is applied to estimate the cohesive Hamaker constants and surface energy components of the materials. The cohesive Hamaker constants range from $85 \mathrm{zJ}$ to $135 \mathrm{zJ}$ across the different films. When these Hamaker constants are compared to prior work using Lifshitz theory and nonpolar probe IGC, the relative magnitudes can be ordered contact angle > Lifshitz $>$ IGC. Furthermore, the dispersive surface energy components estimated here are in good agreement with those estimated by IGC. Due to these results, researchers and technologists will now have access to a comprehensive database of adhesion constants which describe the behavior of these energetic materials over a range of settings.
\end{abstract}

Keywords: Contact Angle;Interfacial Energy; Surface Energy; Wettability; Hamaker Constant; Energetic Materials 


\section{Introduction}

Improved elucidation of the underlying physics which govern the interfacial behavior of explosive materials will enable optimization of explosives detection incheckpoint security applications and improvement in munitions formulation for myriad defense applications[1-4]. However, many of these critical interactions have not been evaluated carefully. Additionally, the relationship between these interactions and the method by which they are characterized has not been examined in detail.Therefore, developing a fundamental knowledge of how various interfacial characterization approaches are related creates a context where results can be appropriately analyzed and utilized. Important to this type of analysis is establishing the Hamaker constant for energetic materials. The adhesiveHamaker constant $\left(A_{i j k}\right)$ determines the magnitude of the van der Waals (vdW) interaction between two materials, $i$ and $k$,interacting in a medium $j[5]$. The cohesive Hamakerconstant $A_{i i}(i . e$, $i=k$ interacting in vacuum), describes thevdW interaction of a material with itself.Furthermore, combining relations exist to estimate the adhesive Hamaker constants using the geometric mean of the cohesive Hamaker constants of the two interacting materials [5]. These cohesive Hamaker constants of solids can be determined via surface energy measurements or using optical and dielectric properties (Lifshitz theory)[5-7].In this way, both inverse gas chromatography (IGC) and contact angle measurements are commonly employed to determine the surface energy of solids.IGC is used to characterize solids in powder form using a series of probe gases with known properties, and contact angle measurements are used to characterize solids with a larger interface using a series of liquids with known properties. A variety of methods can be used to determine surface energy from contact angle measurements. The Owens and Wendt method, which separates surface energy into a polar and dispersive component, will be the focus of this study, as outlined below.

Energetic materials are typically in a powder form, which causes them to not have a large enough interface to perform contact angle measurements.Additionally, the compression of powders for contact angle measurements is not idealgiven the non-trivial effect porosity can have on the contact angle[8]. Additionally, 
crystal faces fracture or deform during compaction, and compaction of sensitive energetics for contact angle measurements can be a safety concern.As a result, 1,3,5-triamino-2,4,6-trinitrobenzene(TATB), an insensitive high explosive, is the only energetic powder that has been compacted for contact angle measurements $[9,10]$. Contact angle measurements have been performed on crystalline pentaerythritoltetranitrate (PETN) (two different crystal faces) and 1,3,5-trinitro-1,3,5-triazacyclohexane (RDX) single crystals, but the experimental details were not reported [11]. The Owens and Wendt method was used to relate the contact angles measured on the RDX in these studies to the surface energy of the RDX [12].IGC experiments have been performed onenergetic materials, including PETN, 2,4,6-trinitrotoluene(TNT), RDX, and1,3,5,7-tetranitro-1,3,5,7tetraazacyclooctane(HMX)[13-16], including arecent study that determined the dispersive surface energy of PETN, TNT, and RDX and related theseto cohesive Hamaker constants[16].In order to overcome the challenges associated with contact angle measurements and to obtain a comprehensive database of adhesion constants, thin films produced by physical vapor deposition will be used for contact angle measurements.

In this work, contact angle values will be evaluated using a family of solvents with varying degrees of polarity on thin films of RDX, TNT, PETN,2,2',4,4',6,6'-hexanitroazobenzene(HNAB), and2,2',4,4',6,6'hexanitrostilbene(HNS) deposited by physical vapor deposition (PVD). These contact angles are then analyzed using the Owens and Wendt method to estimate surface energy components and, ultimately,Hamaker constants. Analyzing five different energetic materials using the same procedure facilitates a direct comparison between materials.Furthermore, comparing cohesive Hamaker constants determined through different approaches elucidates the underlying fundamental interactions accounted for in each approach and, thus, what complementary information can be extracted from these different approaches.

\section{Materials and Methods}

Energetic films of HNAB ( 95\% pure), RDX (with 7-12\%HMX impurity), PETN, HNS, and TNT were fabricated in a custom-designed high-vacuum deposition system. Powders of the various materials were 
loaded into an effusion cell deposition source, and the chamber was evacuated to a base pressure of $\sim 10^{-6}$ Torr (Table S1). The effusion cell was heated to a material-dependent maximum temperature to increase the vapor pressure and deposition rate while preventing substantial decomposition of the explosive materials (Table S1).The deposition substrates were rotated during deposition to promote a uniform thickness distribution.

Several different sample geometries were employed. Each material was initially deposited onto a fused silica substrate that had a thin, water-soluble poly(acrylic acid) (PAA) layer spun-coat on the surface. The samples were then immersed in water to separate the $70-90 \mu \mathrm{m}$ energetic films from the substrates in the form of freestanding films of explosives (the 'lift-off' method[17]). This allowed contact angle experiments to be performed using the smooth interface created against the PAA layer. However, many of the films suffered mechanical failures during this process, and only the TNT films were used. An alternate sample preparation method was required for the other materials. HNAB films were deposited with similar thicknesses on polycarbonate substrates, as these films deposit with a low surface roughness that is largely independent of film thickness[18]. Thin layers $(\sim 5 \mu \mathrm{m})$ of PETN, RDX, and HNS were deposited onto fused silica substrates coated with a thin layer of aluminum. This approximate thickness value was used to ensure that the films were continuous while minimizing surface roughness values (which increases with increasing film thicknessfor these types of material depositions).

Scanning electron microscope (SEM) micrographs of the film surfaces were captured using a Zeiss Supra 35 VP SEM operating at $1.1 \mathrm{kV}$. ABrukerDektakXT profilometerwith a $12.5 \mu \mathrm{m}$ radius stylus was used to create three-dimensional maps to provide surface roughness data. The $2 \mathrm{~mm} \times 2 \mathrm{~mm}$ maps were created by a series of line scans with a $25 \mu \mathrm{m}$ spacing between scans. Contact angle measurements were performed using a Krüss DSA30 Drop Shape Analysis System with DSA4 Software for drop shape analysis. The water used for contact angle measurements was deionized on site and had a resistivity of $\sim 18 \mathrm{M} \Omega \mathrm{cm}$. Ethylene glycol (anhydrous, 99.8\% purity), glycerol (> 99\% purity), and diiodomethane (containing a copper stabilizer, 99\% 
purity) were purchased from Sigma-Aldrich and used as received. Liquid drops with volume between 1 and 3 $\mu \mathrm{Lwere}$ suspended from a dosing needle andsubsequently brought into contact with the films.Each static contact angle measurement was performed at previously untested locations on each film. Measurements were made at $\sim 28 \%$ relative humidity.

\section{Theory}

A number of considerations must be established while measuring and analyzing data collected from contact angle measurements. For instance, contact angle measurements can be related to the surface energy of the material through thecommonly-used Young's Equation

$$
\gamma_{l v} \cos (\theta)=\gamma_{s v}-\gamma_{s l}
$$

Here, $\theta$ is the contact angle,and $\gamma_{l v}, \gamma_{s v}$, and $\gamma_{s l}$ are the interfacial energies at the liquid-vapor, solid-vapor, and solid-liquid interfaces. Figure 1 illustrates this equilibrium relationshipbetween the three interfacial energies. The contact angle is, ideally, a thermodynamic constant; however, in practicethis tends to not be the casedue to surface roughness, chemical heterogeneity, adsorption, absorption, dissolution, and/or other chemical interactions at the liquid-solid interface[19-22].Droplet size can influence the contact angle as well[23-25]. If a droplet is too small, line tension influences the apparent contact angle. On the other hand, if the droplet is too large, gravity influences the apparent contact angle. Furthermore, the input of mechanical energy from either a falling drop or a drop injected directly on the surface will also influence drop shape and should be avoided.As such, great care must be employed whenperforming the contact angle measurements in order to ensure proper results. This was done by selecting compatible test liquids, performing multiple measurements, and following the deposition procedure previously described. 


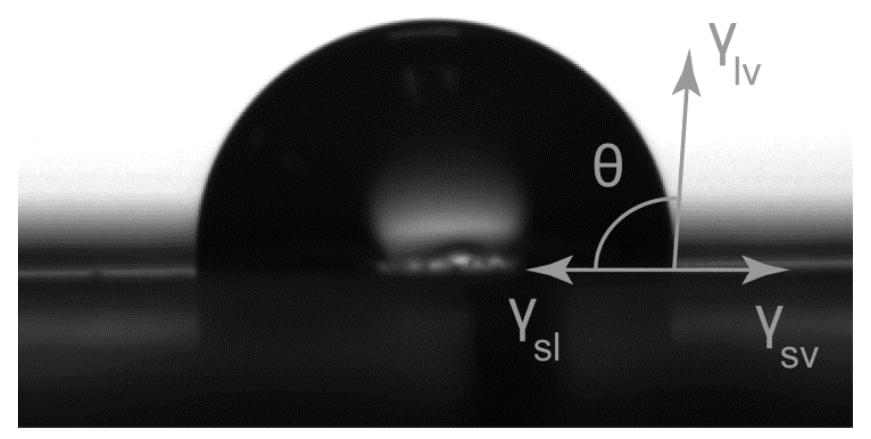

Figure 1. A droplet of water on a trinitrotoluene (TNT) thin film illustrating the components of Young's equation. In this system, $\theta$ is the contact angle, and $\gamma_{l v}, \gamma_{s v}$, and $\gamma_{s l}$ are the interfacial energies at the watervapor, TNT-vapor and TNT-water interfaces. The contact angle value is greater than $90^{\circ}$, in agreement with the relatively hydrophobic nature of the TNT thin film.

A variety of approaches have been developed to determine the surface energy of solids from contact angle measurements.No single approach has been determined to be best for all cases, and the results will be dependent, in part, on the analysis technique selected [26]. A majority ofanalytical techniques implementFowkes' basic postulate, which states that surface energy can be separated into distinct additive components [27]. This basic postulate is not rigorously true as surface energy cannot be rigorously separated into unique components specific to different interaction types [19].Regardless, it is an acceptable approximation and isthe underlying assumption of all prominent contact angle analysis methods, including the Owens and Wendt method[19]. The Owens and Wendt method, which is commonly applied to low surface energy materials (as is the case of the materials of study here), will be utilized in this effort [28-30].In this method, the surface energy is assumed to be comprised of two components.

$$
\gamma=\gamma^{d}+\gamma^{p}
$$

In Equation 2, the superscripts $d$ and $p$ denote the dispersive and polar components of the surface energy, respectively. The dispersive component is used to represent London-dispersion forces and the polar component is used to represent all other forces present. These forcesinclude theKeesom, Debye, and hydrogen-bonding 
forces.Additionally, the Owens and Wendt method accounts for any site-specific interaction. The components of the solid and liquidare then assumed to interact via a geometric mean, as shown in Equation 3.

$$
\gamma_{s l}=\gamma_{s v}+\gamma_{l v}-2 \sqrt{\gamma_{s}^{d} \gamma_{l}^{d}}-2 \sqrt{\gamma_{s}^{p} \gamma_{l}^{p}}
$$

This assumption has not been validated for the dispersive or polar components of the energy[20,31,32]. Nevertheless, this assumption is employed as no better alternative has been demonstrated. The substitution of Equations 2 and 3 into Equation1 (and subsequent rearrangement) yields Equation 4.

$$
\frac{(\cos (\theta)+1) \gamma_{l v}}{2 \sqrt{\gamma_{l}^{d}}}=\sqrt{\gamma_{s}^{p}}\left(\frac{\sqrt{\gamma_{l}^{p}}}{\sqrt{\gamma_{l}^{d}}}\right)+\sqrt{\gamma_{s}^{d}}
$$

Therefore, by performing contact angle measurements using multiple liquids with known surface energies, including the polar and dispersive components, $\gamma_{s}^{p}$ and $\gamma_{s}^{d}$ are the only remaining unknown parameters. As such, appropriate plotting of Equation 4can lead to their evaluation through a simple linear regression. The surface energies of the liquids used in this study are shown in Table 1. In some occasions, surface energies of solids determined from contact angle measurements have been found to be dependent on the test liquids selected for contact angle measurements. For this reason, multiple test liquids should be employed and the liquids should remain constant for all materials that will be compared.

Table 1. Values of the polar and dispersivecomponents of thesurface energy of the liquids used for the contact angle measurements in this effort

\begin{tabular}{cccc}
\hline & \multicolumn{2}{c}{$\begin{array}{c}\text { Surface Energy } \\
\text { Components }\left[\mathbf{m J} / \mathbf{m}^{2}\right]\end{array}$} & \\
\cline { 2 - 3 } Liquid & $\gamma_{l}^{d}$ & $\gamma_{l}^{p}$ & Ref. \\
\hline Water & 21.8 & 51 & {$[27]$} \\
Glycerol & 21.2 & 41.5 & {$[33]$} \\
Ethylene Glycol & 30.9 & 16.8 & {$[34]$} \\
Diiodomethane & 50.8 & 0 & {$[34]$} \\
\hline
\end{tabular}


The solid surface energy can be used to estimate the cohesiveHamaker constant of a materialusing the following relationship.

$$
A_{i i}=24 \gamma_{s} D_{0}^{2}
$$

Here, $D_{0}$ is the "cut-off" separation distance,and it is approximately $0.165 \mathrm{~nm}[5]$.This relationship breaks down for materials with significant hydrogen bonding, which does not include any of the energetic materials of this study; therefore, this approximation is useful for this particular effort.

\section{Results and Discussion}

\section{Contact Angle Measurements}

The contact angle valuesreported in Table 2 are representative of the equilibrium contact angle between the pure liquid and solid surface.Three drops were measured for each solid/liquid pair unless otherwise noted. Multiple images were captured, measured, and averaged to determine the contact angle for each drop. Measurements were taken immediately after drop deposition with the exception of glycerol drops, which were allowed up to 60 seconds to reach an equilibrium value; this extra time was afforded to glycerol due to the relatively high viscosity ( $1500 \mathrm{cP}[35])$ of this fluid.If a liquid other than glycerolbegan to spread immediately after deposition, the measurement was not considered to represent the equilibrium contact angle. 
Table 2. Average contact angle values measured for test liquids on thin films of energetic materials. All measurements were performed in triplicate unless otherwise noted. The error indicates the standard deviation.

\begin{tabular}{lcccl}
\hline \multirow{2}{*}{$\begin{array}{l}\text { Energetic } \\
\text { Material }\end{array}$} & \multicolumn{4}{c}{ Test Liquid Contact Angle Values [ ${ }^{\circ}$} \\
\cline { 2 - 5 } & Water & Glycerol & $\begin{array}{c}\text { Ethylene } \\
\text { Glycol }\end{array}$ & Diiodomethane \\
\hline PETN & Spread $^{\mathrm{a}}$ & $59.5 \pm 1.2$ & Spread $^{\mathrm{a}}$ & $48.0 \pm 3.0$ \\
TNT & $94.5 \pm 1.8$ & $75.1 \pm 1.9$ & $53.8 \pm 0.7$ & $38.0 \pm 3.3$ \\
RDX & $75.8 \pm 1.0$ & $61.3 \pm 1.4$ & $38.3 \pm 0.9$ & $18.7 \pm 1.1$ \\
HNAB & $97.8 \pm 0.8$ & $77.6 \pm 1.4$ & $57.3 \pm 1.0$ & $17.8 \pm 3.7^{\mathrm{b}}$ \\
HNS & $97.1 \pm 0.3$ & $66.4 \pm 1.1$ & $36.7 \pm 0.9$ & Wet \\
\hline
\end{tabular}

a. The drop began to spread immediately after it was deposited on the energetic film.

b. Four measurements

Surface roughness should be minimized for the contact angle measurements to be most representative of the equilibrium contact angle value, because surface features can cause deviationsin the measured contact angles. Figure 2 shows the SEM images of the energetic films studied. The RMSroughness values of the films (obtained from $2 \mathrm{~mm} \times 2 \mathrm{~mm}$ profilometermaps) range from 75 to $1,700 \mathrm{~nm}$ (Table S2). Due to the rather uneven topological features of these films across multiple length scales, the exact surface roughness values cannot be accurately captured in full.Therefore, these roughness values should be regarded as estimates. The minor deviations in contact angle measurements are attributed to these features. 


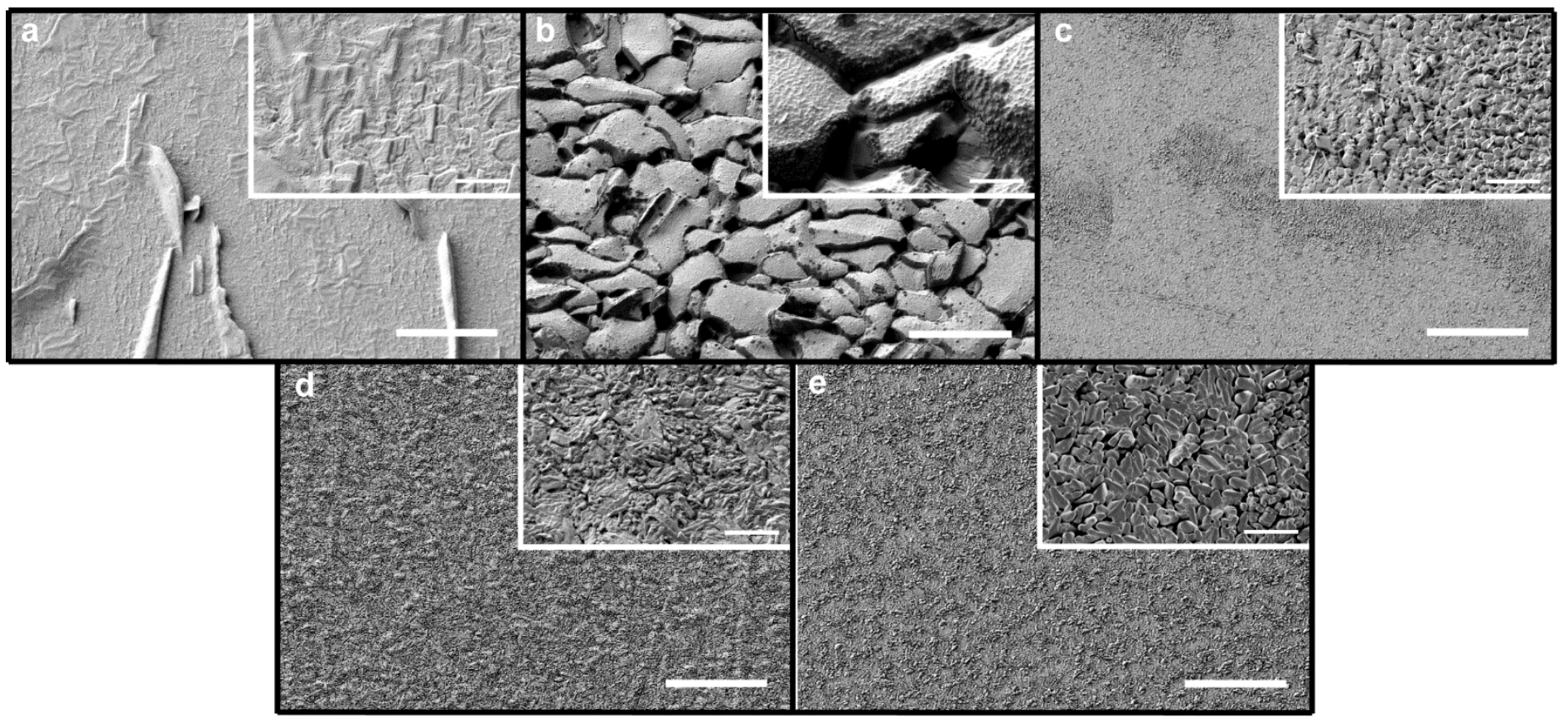

Figure 2. SEM images of:(a) PETN,(b) TNT,(c) RDX,(d) HNAB, and (e) HNS. The scalebars for each of the images represent $20 \mu \mathrm{m}$ and the scalebars of each of the inset images represent $2 \mu \mathrm{m}$. The morphology varies substantially from film type to film type.This is due to the deposition conditions and the different microstructures innate to the materials[18,36]. The minimal roughness values of the films contributes to the minor distribution of contact angle measurements observed and deviation from the thermodynamic equilibrium contact angle.

The contact angles measured for the RDX and PETN films differ from previously performed contact angle measurements on single crystals by $1.5^{\circ}$ to $17^{\circ}[11]$. A number of explanations for this are possible; however, the exact explanation cannot be determined due to the lack of experimental detail available from these previous reports.Additionally, the two PETN single crystal facesreported in the literaturehad contact angles that differedby $5^{\circ}$ to $10^{\circ}[11]$. This dissimilarity, in addition to the lack of experimental details, reduces the concern regarding the variation of observed contact angles between studies.

\section{Surface Energy}

The contact angle values for each drop (Table 2) and the surface energy components of the test liquids (Table 1) were used to determine the different surface energy components related to the thin films of energetic materials. A linear regressionwas performed with a95\% confidence interval of regression coefficients. All 
slopes were positive, however, the slope of the lines (i.e., the values related to the $\sqrt{\gamma_{s}^{p}}$ term of Equation 4) for HNS and HNAB were less than 1. The polar componentvalues of these filmswere, therefore,considered negligible and another analysis was performed using a constant regression modelwith a 95\% confidence interval of regression.Figure 3 shows the different surface energy components of the six energetic thin films. The dispersive surface energy component for all films dominates the total surface energy.Importantly, the dispersive components predicted using the IGC and contact angle measurements are in agreement. This suggests that the PVDfilm interfaces are similar to the crystal faces found in the powders and that the same intermolecular interactions are characterized. Using these determined surface energy components, wetting envelopes can be generated (Figure S1). The wetting envelopes of the different energetic films will assist in the prediction of which liquids (with known surface energy components) will wet the energetic films[37]. RDX had a similar overall surface energy to prior work $\left(\gamma^{d}=37.3 \mathrm{~mJ} / \mathrm{m}^{2}, \gamma^{p}=13.0 \mathrm{~mJ} / \mathrm{m}^{2}, \gamma=50.3 \mathrm{~mJ} / \mathrm{m}^{2}\right)$ using the same method with different contact angle measurements[12].

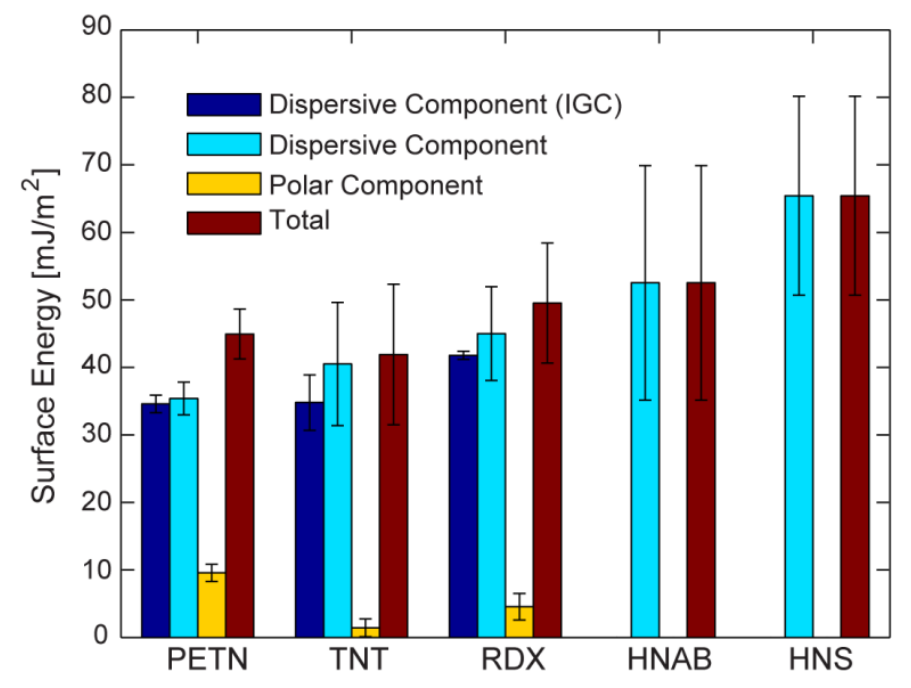

Figure 3. Surface energy and surface energy components of the energetic materials. The dispersive surface energy components from this study compare well to the dispersive surface energy determined through the IGC method[16]. The dispersive component dominates for each of the films studied. The error bars for the results of this work indicate the $95 \%$ confidence interval of regression coefficients. The IGC dispersive component error bars indicate a $95 \%$ confidence interval around the mean values. 


\section{Cohesive Hamaker Constants}

Equation 6 was used to estimate the Hamaker constants of the materials from the measured surface energy values (Figure 4). The PETN, RDX and TNT results of both this analysis and the IGC analysisare within the error of the Hamaker constants determined via Lifshitz theory[16]. TheLifshitz-predicted Hamaker constantsare consistently higher than the IGC-predicted Hamaker constantsas the IGC analysis does not take into account the polar surface energy component in the interaction. The contact angle-predicted Hamaker constants are consistently higher than the Lifshitz-predicted Hamaker constants due to the inclusion of hydrogen bonding interactions in the polar surface energy components of the contact angle-predicted Hamaker constants.This is the first time HNAB or HNS have been analyzed;it is expected that the HNAB and HNS results will agree well with a Lifshitz theory analysis as the polar component (and therefore hydrogen-bonding) was found to be negligible.

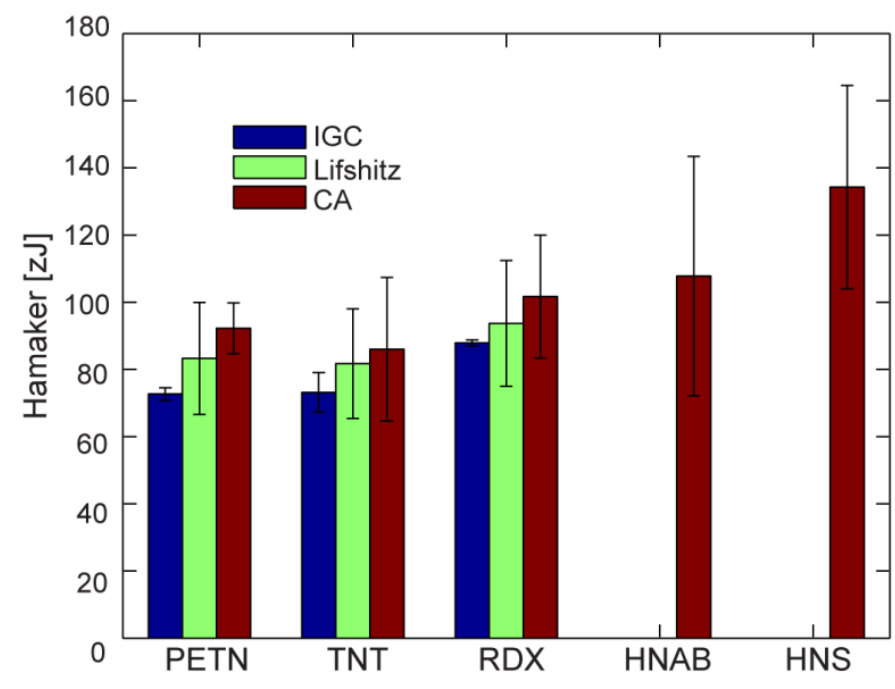

Figure 4. Cohesive Hamaker constant comparison for IGC[16], Lifshitz[16], and contact angle (CA) approaches. Both the IGC and contact angle predictionsare within the error of the Lifshitz prediction; however, a clear trend is apparent. The LifshitzHamaker constant is underestimated by IGC and overestimated by contact angle analysis. The IGC only accounts for London dispersion forces, whereas the Lifshitz theory accounts for London dispersion forces, Keesom forces, and Debye forces. The contact angle approach accounts for not only London dispersion forces, Debye forces, and Keesom forces but also hydrogen-bonding forces. The dispersive component dominates for each film studied, and this is why an agreement is observed across the different approaches. The contact angle (CA) error bars indicate the $95 \%$ confidence interval of regression coefficients. The Lifshitz error bars indicate a $20 \%$ confidence. The IGC error bars indicate a $95 \%$ confidence interval around the mean. 


\section{Conclusions}

The cohesive Hamaker constant and surface energy of five explosives have been determined through contact angle measurements. The cohesive Hamaker constants of the five explosive material films determined in this study range from 85 to $135 \mathrm{zJ}$. This demonstrates that cohesive Hamaker constants of nitro-containing explosives do not vary substantially across the range of possible Hamaker constants $\left(\sim 40<A_{i i}<400 \mathrm{zJ}\right)[5]$. Although different aspects of HNAB and HNS have been well-characterized elsewhere[38-40], this is the first study characterizing their interfacial properties. Their polar surface energy components were found to be negligible, meaning the contributions of the Keesom and Debye interactions to the vdW forces are negligible and the overall surface energy of the energetic materials is primarily composed of the dispersive surface energy component.

The contact angle results for PETN, TNT, and RDX results were compared to adhesion constants previously determined using IGC and Lifshitz theory [16]. Through this comparison, a description of the fundamental intermolecular interactions considered in each method is elucidated. The dispersive surface energy components of PETN, TNT, and RDX agree with previous IGC results indicating that both studies depend primarily upon the same intermolecular interaction, Londondispersion forces. Agreement of surface energy components across the contact angle and IGC methods has been shown before with other materials [41]. For cohesive Hamaker constants, contact angle measurement analysis was found to over-predict the value compared to Lifshitz theory results due to the inclusion of hydrogen bonding forces. Dispersive probe IGC underestimates the Lifshitz cohesive Hamaker constant as it does not include Keesom and Debye interactions. Historically, Hamaker constants have been readily employed regardless of origin [5]. In the future, the origin of the Hamaker constant, and therefore which intermolecular forces it represents, can be taken into consideration when Hamaker constant values are used in different settings. Comparing the results of these three complementary approaches 
offers insight into the components that control adhesion and provides a comprehensive database of constants which describe the adhesion behavior in a variety of settings.

\section{Acknowledgements}

We thank Michael Marquez (deposition) and M. Barry Ritchey (SEM) for their assistance with the noted experimental efforts. Sandia National Laboratories is a multi-program laboratory managed and operated by Sandia Corporation, a wholly owned subsidiary of Lockheed Martin Corporation, for the U.S. Department of Energy's National Nuclear Security Administration under contract DE-AC04-94AL85000. The work of the Sandia team was supported in part by the Joint Department of Defense/Department of Energy Munitions Technology Development Program. The work of D.J.H., B.W.B., and S.P.B. at Purdue University was supported by the U.S. Department of Homeland Security, Science and Technology Directorate, Office of University Programs, under Grant Award 2013-ST-061-ED0001. The views and conclusions contained in this document are those of the authors and should not be interpreted as necessarily representing the official policies, either expressed or implied, of the U.S. Department of Homeland Security. We gratefully acknowledge this support.

\section{References}

[1] J.R. Verkouteren, J.L. Coleman, R.A. Fletcher, W.J. Smith, G.A. Klouda, G. Gillen, A method to determine collection efficiency of particles by swipe sampling, Meas. Sci. Technol. 19 (2008) 115101.

[2] S.J.P. Palmer, Adhesion Studies between HMX and EDC37 Binder System, AIP Conf. Proc. 845 (2006) 917-920.

[3] D.M. Williamson, N.R. Hamilton, S.J.P. Palmer, a P. Jardine, C. Leppard, Thermodynamic work of adhesion measurements of polymer bonded explosive constituents via the Wilhelmy plate technique and their application to AFM pull-off experiments, J. Phys. Conf. Ser. 500 (2014) 112068.

[4] J.D. Yeager, K.J. Ramos, S. Singh, M.E. Rutherford, J. Majewski, D.E. Hooks, Nanoindentation of explosive polymer composites to simulate deformation and failure, Mater. Sci. Technol. 28 (2012) 11471155.

[5] J.N. Israelachvili, Van der Waals Forces between Particles and Surfaces, in: Intermol. Surf. Forces Revis. Third Ed., 2011: pp. 253-289. doi:10.1016/B978-0-12-375182-9.10013-2.

[6] E.M. Lifshitz, The theory of Molecular Attractive Forces between Solids, Sov. Physics, JETP. 2 (1956) 73-83.

[7] V.A. Parsegian, Van der Waals Forces : a Handbook for Biologists, Chemists, Engineers, and Physicists, 
Cambridge University Press, New York, 2006.

[8] J. Litster, B. Ennis, The Science and Engineering of Granulation Processes, Springer Netherlands, Dordrecht, 2004. doi:10.1007/978-94-017-0546-2.

[9] J. Sharma, B.C. Beard, M. Chaykovsky, Correlation of impact sensitivity with electronic levels and structure of molecules, J. Phys. Chem. 95 (1991) 1209-1213.

[10] T. Rivera, M.L. Matuszak, Surface properties of potential plastic-bonded explosives (PBX), J. Colloid Interface Sci. 93 (1983) 105-108.

[11] W.L. Elban, Surface Energies of High Explosives PETN and RDX from Contact Angle Measurements, J. Mater. Sci. 14 (1978) 1008-1011.

[12] J.D. Yeager, K.J. Ramos, D.E. Hooks, J. Majewski, S. Singh, Formulation-Derived Interface Characteristics Contributing to Failure in Plastic-Bonded Explosive Materials, in: 15th Int. Detonation Symp., 2014.

[13] Y. Luo, M. Du, The Use of Inverse Gas Chromatography (IGC) to Determine the Surface Energy of RDX, Propellants, Explos. Pyrotech. 32 (2007) 496-501.

[14] U. Teipel, Energetic materials: particle processing and characterization, John Wiley \& Sons, Inc., 2006.

[15] J.K. Bower, J.R. Kolb, C.O. Pruneda, Polymeric Coatings Effect on Surface Activity and Mechanical Behavior of High Explosives, Ind. Eng. Chem. Prod. Res. Dev. 19 (1980) 326-329.

[16] A.J. Harrison, A. Otte, T. Carvajal, R. Pinal, S.P. Beaudoin, Cohesive Hamaker Constants and Dispersive Surface Energies of RDX, PETN, TNT, and Ammonium Nitrate-Based Explosives, Propellants, Explos. Pyrotech. (2015) n/a-n/a.

[17] V. Linder, B.D. Gates, D. Ryan, B.A. Parviz, G.M. Whitesides, Water-soluble sacrificial layers for surface micromachining., Small. 1 (2005) 730-6. doi:10.1002/smll.200400159.

[18] R. Knepper, K. Browning, R.R. Wixom, A.S. Tappan, M.A. Rodriguez, M.K. Alam, Microstructure Evolution during Crystallization of Vapor-Deposited Hexanitroazobenzene Films, Propellants, Explos. Pyrotech. 37 (2012) 459-467.

[19] D.Y. Kwok, A.W. Neumann, Contact angle measurement and contact angle interpretation, Adv. Colloid Interface Sci. 81 (1999) 167-249. doi:10.1016/S0001-8686(98)00087-6.

[20] J. Kloubek, Development of methods for surface free energy determination using contact angles of liquids on solids, Adv. Colloid Interface Sci. 38 (1992) 99-142.

[21] A. Rudawska, E. Jacniacka, Analysis for determining surface free energy uncertainty by the OwenWendt method, Int. J. Adhes. Adhes. 29 (2009) 451-457.

[22] M. Morra, E. Occhiello, F. Garbassi, Knowledge about polymer surfaces from contact angle measurements, Adv. Colloid Interface Sci. 32 (1990) 79-116. doi:10.1016/0001-8686(90)80012-O.

[23] R.J. Good, M.. Koo, The effect of drop size on contact angle, J. Colloid Interface Sci. 71 (1979) 283292.

[24] J. Gaydos, A.. Neumann, The dependence of contact angles on drop size and line tension, J. Colloid Interface Sci. 120 (1987) 76-86.

[25] D. Li, A.W. Neumann, Determination of line tension from the drop size dependence of contact angles, Colloids and Surfaces. 43 (1990) 195-206. doi:10.1016/0166-6622(90)80288-F.

[26] M. Żenkiewicz, Comparative study on the surface free energy of a solid calculated by different methods, 
Polym. Test. 26 (2007) 14-19.

[27] F.M. Fowkes, Attractive Forces at Interfaces, Ind. Eng. Chem. 56 (1964) 40-52.

[28] D.K. Owens, R.C. Wendt, Estimation of the surface free energy of polymers, J. Appl. Polym. Sci. 13 (1969) 1741-1747.

[29] D.H. Kaelble, Dispersion-Polar Surface Tension Properties of Organic Solids, J. Adhes. 2 (1970) 66-81.

[30] W. Rabel, Wetting theory and its application to the study and use of the surface properties of polymers, Farbe Und Lack. 77 (1971) 997-1005.

[31] F.M. Fowkes, M.B. Kaczinski, D.W. Dwight, Characterization of polymer surface sites with contact angles of test solutions. 1. Phenol and iodine adsorption from methylene iodide onto PMMA films, Langmuir. 7 (1991) 2464-2470.

[32] S. Chevalier, M.K. Chaudhury, Further Reflection on the Geometric Mean Combining Rule for Interfacial Tension, Langmuir. (2015) 150921125608004.

[33] H.J. Busscher, A.W.J. van Pelt, P. de Boer, H.P. de Jong, J. Arends, The effect of surface roughening of polymers on measured contact angles of liquids, Colloids and Surfaces. 9 (1984) 319-331.

[34] G. Ström, M. Fredriksson, P. Stenius, Contact angles, work of adhesion, and interfacial tensions at a dissolving Hydrocarbon surface, J. Colloid Interface Sci. 119 (1987) 352-361.

[35] M.L. Sheely, Glycerol Viscosity Tables, Ind. Eng. Chem. 24 (1932) 1060-1064.

[36] R. Knepper, A.S. Tappan, R.R. Wixom, M.A. Rodriguez, Controlling the microstructure of vapordeposited pentaerythritol tetranitrate films, J. Mater. Res. 26 (2011) 1605-1613.

[37] D. Janssen, R. De Palma, S. Verlaak, P. Heremans, W. Dehaen, Static solvent contact angle measurements, surface free energy and wettability determination of various self-assembled monolayers on silicon dioxide, Thin Solid Films. 515 (2006) 1433-1438.

[38] J.-S. Lee, C.-K. Hsu, C.-L. Chang, A study on the thermal decomposition behaviors of PETN, RDX, HNS and HMX, Thermochim. Acta. 392-393 (2002) 173-176. doi:10.1016/S0040-6031(02)00099-0.

[39] W. Gui-xiang, S. Chun-hong, G. Xue-dong, X. He-ming, Theoretical Investigation on Structures, Densities, Detonation Properties, and the Pyrolysis Mechanism of the Derivatives of HNS, J. Phys. Chem. A. 113 (2009) 1318-1326. doi:10.1021/jp804950z.

[40] M.A. Rodriguez, C.F. Campana, A.D. Rae, E. Graeber, B. Morosin, Form III of 2,2',4,4',6,6'hexanitroazobenzene (HNAB-III), Acta Crystallogr. Sect. C Cryst. Struct. Commun. 61 (2005) o127o130. doi:10.1107/S0108270105000569.

[41] S.K. Papadopoulou, G. Dritsas, I. Karapanagiotis, I. Zuburtikudis, C. Panayiotou, Surface characterization of poly(2,2,3,3,3-pentafluoropropyl methacrylate) by inverse gas chromatography and contact angle measurements, Eur. Polym. J. 46 (2010) 202-208. doi:10.1016/j.eurpolymj.2009.11.002. 


\section{Graphical Abstract}
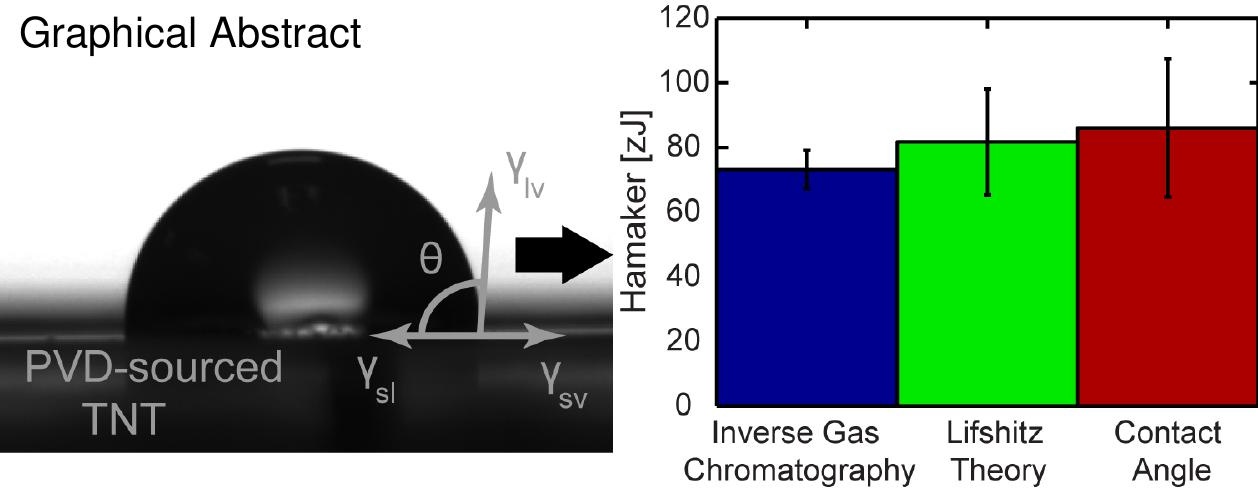